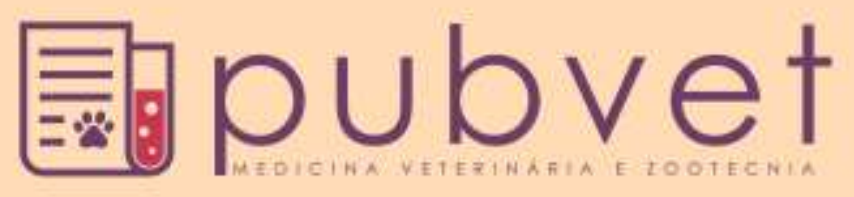

HTTP://DX.DOI.ORG/10.22256/PUBVET.V11N3.298-305

\title{
Digestibilidade e contribuição da cecotrofia de coelhos alimentados com ou sem óleo vegetal na dieta
}

\author{
Eriane de Paula ${ }^{1 *}$, Walter Motta Ferreira ${ }^{2}$, Felipe Norberto Alves Ferreira ${ }^{3}$, Martolino \\ Barbosa da Costa Júnior ${ }^{4}$, Katiuscia Cristina das Neves Mota ${ }^{5}$, Leonardo Francisco da \\ Rocha $^{6}$
}

1Doutora em zootecnia pela Universidade Federal de Minas Gerais Belo Horizonte -MG Brasil. E-mail: Erianep@hotmail.com

${ }^{2}$ Professor da Universidade Federal de Minas Gerais, Departamento de Zootecnia. Belo Horizonte-MG Brasil. E-mail: mottafer@yahoo.com ${ }^{3}$ Doutorando em Zootecnia pela Universidade Federal de Minas Gerais, Departamento de Zootecnia. Belo Horizonte-MG Brasil. E-mail: felipe.norberto@yahoo.com.br

${ }^{4}$ Doutorando em Zootecnia pela Universidade Federal de Minas Gerais, Departamento de Zootecnia. Belo Horizonte-MG Brasil. E-mail: martolino@zootecnista.com.br

${ }^{5}$ Mestranda em pela Universidade Federal de Minas Gerais, Departamento de Zootecnia. Belo Horizonte-MG Brasil. E-mail: katiusciazootecnia@hotmail.com

Mestranda em pela Universidade Federal de Minas Gerais, Departamento de Zootecnia. Belo Horizonte-MG Brasil. E-mail: leozootecniaufla@yahoo.com.br

RESUMO. O experimento foi realizado no Laboratório de Metabolismo e Nutrição Animal localizado na Escola de Veterinária da Universidade Federal de Minas Gerais (EVUFMG). Foram utilizados 20 coelhos da raça (Nova Zelândia branco), de ambos os sexos, com 63 dias de idade. Os animais foram distribuídos em um desenho inteiramente casualizado, constituído por dois tratamentos, duas rações experimentais (T1: ração sem adição de óleo vegetal e T2: ração com parte da energia complementada com óleo vegetal) e dez repetições. As fezes de cada animal foram coletadas em sua totalidade, uma vez ao dia, pela manhã. Foram avaliados os coeficientes de digestibilidade aparente da matéria seca (CDMS), proteína bruta (CDPB), extrato etéreo (CDEE), da matéria orgânica (CDMO) e da energia bruta (CDEB) das rações. A ração sem adição de óleo apresentou uma maior CDEB 61,34\% e menor CDMS 60,95\%. Os outros parâmetros avaliados não demonstraram diferenças entre os tratamentos. Foi avaliada a composição e a produção dos cecotrófos dos coelhos alimentados com as dietas já mencionadas. Em relação à composição dos cecotrófos não foram observadas diferenças na MS e na PB pelas diferentes dietas oferecidas, todavia, o teor de MO e EE dos mesmos foram diferentes sendo, que a ração sem adição de óleo apresentou cecotrófos com maiores teores de $\mathrm{MO}$ e menor teor em EE. Com relação à contribuição nutricional dos nutrientes dos cecotrófos, a CNMS e a CNEE houve diferenças estatísticas significativas, sendo que a ração complementada com lipídios proporcionou uma maior CNMS 23,01\% vs 17,83\% da dieta não complementada; e uma menor CNEE 9,65\% vs $15,17 \%$ da dieta não complementada. As dietas não ocasionaram diferenças na produção dos cecotrófos. Com base nos resultados obtidos concluiu-se ser possível a inclusão de óleo vegetal nas dietas de coelhos em crescimento no nível de $6 \%$, desde que respeitado as recomendações de fibra estabelecida na literatura, quando da confecção da dieta.

Palavras chaves: Coelhos, cecotrófos, digestibilidade, óleo vegetal

\section{Digestibility and contribution of caecotrophy of rabbits fed with or without vegetable oil in the diet}

ABSTRACT. The experiment was conducted at Animal Metabolism and Nutrition Laboratory located at the Veterinary School of the Federal University of Minas Gerais (VSUFMG). 20 rabbits (Oryctolagus cuniculus), race (New Zealand White) were used, of both 
sexes, with 63 days old. The animals were distributed in a completely randomized design, consisting of two treatments, two experimental diets (T1: diet without the addition of vegetable oil vegetable and T2: diet complemented with oil vegetable) and ten repetitions. The feces of each animal were collected daily in the morning. We evaluated the apparent digestibility of dry matter (CDDM), crude protein (CDCP), Ethereal Extract (CDEE), organic matter (CDOM) and gross energy (CDGE) of the feed. A diet with carbohydrate as the main source of energy had a higher CDGE 61.34\% and lower CDMS 60.95\%. The other parameters evaluated showed no differences between treatments. It evaluated the composition and production of cecotrophes rabbits fed with the aforementioned diet. Regarding the composition of cecotrophes no differences were observed in dry matter and $\mathrm{CP}$ by different diets offered, however, the $\mathrm{OM}$ and $\mathrm{EE}$ content were different and that a diet without lipid complementation showed cecotrophes higher OM content and lower content EE. Regarding the nutritional contribution of nutrients from cecotrophes, the CNDM and CNEE were no significant differences, and the diet supplemented with lipids provided greater CNDM $23.01 \%$ vs $17.83 \%$ of the diet not complementation and a smaller CNEE $9.65 \%$ vs $15.17 \%$ of the diet not complementation. The diets did not result in differences in the production of cecotrophes. Based on the results obtained it was concluded be possible inclusion of vegetable oil in the diets of growing rabbits in the $6 \%$ level, provided that respect the limits established in the literature fibers, when making the diet.

Keywords: Carbohydrates, digestibility, caecotrophy, rabbits, and vegetable oil

\section{Digestibilidad y contribución de cecotrofia de conejos alimentados con o sin aceite vegetal en la dieta}

RESUMEN. El experimento se llevó a cabo en el Laboratorio de Metabolismo y Nutrición Animal localizado en la Escuela de Veterinaria de la Universidad Federal de Minas Gerais (UFMG-EV). Fueron utilizados 20 conejos de la raza Nueva Zelanda blancos (hembras y machos), con 63 días de edad. Los animales se distribuyeron en un diseño completamente al azar, constituido por dos tratamientos, dos dietas experimentales (T1: dieta sin adición de aceite vegetal y T2: ración con un parte de la energía suplementada con aceite vegetal) y diez repeticiones. Las heces de cada animal se colectaron en su totalidad, una vez al día, por la mañana. Fueron evaluados los coeficientes de digestibilidad aparente de la materia seca (CDMS), proteína cruda (CCPE), extracto etéreo (EEDC), materia orgánica (CDMO) y energía bruta (CDEB) de las raciones. La dieta sin adición de aceite mostró mayor CDEB $61,34 \%$ y menor CDMS 60,95\%. Los otros parámetros evaluados no presentaron diferencias entre los tratamientos. Se evaluó la composición y producción de cecótrofos de los conejos alimentados con las dietas ya mencionadas. En cuanto a la composición de los cecótrofos no se observaron diferencias de la MS y PB entre las diferentes dietas que se ofrecieron, sin embargo, el MO y el contenido lipídico de los mismos fue diferentes, siendo que, la ración sin adición de aceite mostró cecótrofos con mayor contenido de MO y menor contenido de EE. En cuanto a la contribución nutricional de los cecótrofos, el CNMS y CNEE hubo diferencias estadísticamente significativas, siendo que la ración suplementada con lípidos proporcionó mayor CNMS $23,01 \%$ frente a $17,83 \%$ de la dieta no suplementada; y un menor CNEE 9,65\% frente a 15,17\% de la dieta no suplementada. Las dietas no ocasionaron diferencias en la producción de cecótrofos. Con base a los resultados obtenidos se concluyó ser posible la inclusión de aceite vegetal en la alimentación de conejos en crecimiento en un nivel de 6\%, siempre que se cumplan con las recomendaciones de fibra establecidos en la literatura, al formular la dieta.

Palabras clave: conejos, cecótrofos, digestibilidad, aceite vegetal

\section{Introdução}

O coelho (Oryctolagus cuniculus) é um animal monogástrico de ceco funcional que realiza a cecotrofia que possibilita a ingestão de material altamente fibroso com sua concomitante fermentação no ceco, e transformação em cecotrófo. Os cecotrófos são para o coelho um 
alimento muito nutritivo, que satisfaz parte de sua exigência nutricional diária, portanto quaisquer alimentos ou dieta que possa vir a interferir nesse comportamento ou composto devem ser avaliados.

Os coelhos não possuem a necessidade da incorporação de ácidos graxos em sua dieta. Todavia a incorporação de lipídios a dieta é uma forma de aumentar a densidade energética da mesma, e isso se faz justificável sobre tudo quando ela é rica em fibras como no caso das cunículas e em animais na fase de crescimento que demandam uma alta quantidade de energia. Os coelhos regulam a sua ingestão de alimento em resposta à concentração energética da ração, portanto um aumento indiscriminado na concentração de energia da dieta pode resultar em mudanças marcantes no consumo de ração, na conversão alimentar, na composição da carcaça e na qualidade dos pellets produzido (Maertens, 1998, Pascual et al., 1999, Xiccato, 1996).

A influência da inclusão de lipídios a dieta e sua ação sobre a digestibilidade da fibra e da proteína não é muito clara e os resultados são conflitantes (Fortun-Lamothe, 1997). Ferreira et al. (2006) não encontraram influências sobre a digestibilidade dos nutrientes quando da adição de lipídios a dieta de coelhos, exceto sobre o EE e FDN.

De acordo com Fernández-Carmona et al. (2000) e Xiccato (1999) a inclusão de lipídios a dietas de coelhos aumenta o seu conteúdo de energia digestível e reduz a ingestão de alimento, sem, contudo afetar o crescimento animal ou quando afeta esse é favorável, isso acarreta melhores índices de conversão alimentar.

A composição nutricional dos cecotrófos é influenciada pela dieta, assim os mesmos podem ter uma maior ou menor riqueza de nutrientes. O consumo de cecotrófos é influenciado pela proteína e pela energia da dieta. Quando a dieta possui uma baixa quantidade de energia, a ingestão dos cecotrófos é maximizada (Jenkins, 1999).

Objetivou-se com este trabalho avaliar a digestibilidade dos nutrientes de dietas complementadas ou não com óleo vegetal, assim como seu efeito na produção e composição dos cecotrófos.

\section{Material e Métodos}

O experimento foi realizado no Laboratório de Metabolismo e Nutrição Animal situado nas dependências do Departamento de Zootecnia da Escola de Veterinária da Universidade Federal de Minas Gerais - UFMG.

Foram utilizados 20 coelhos (Oryctolagus cuniculus) da raça Nova Zelândia branco, de ambos os sexos, com 63 dias de idade. Os animais foram pesados, alojados individualmente em gaiolas de metabolismo fabricadas com arame galvanizado com dimensões $40 \times 60 \times 45 \mathrm{~cm}$ (comprimento, largura e altura), providas de bebedouros tipo (tipo chupeta) e comedouros semiautomáticos. Os animais foram distribuídos de acordo com um delineamento experimental inteiramente casualizado, constituído por dois tratamentos, duas rações experimentais ( $\mathrm{T} 1$ ração com carboidratos na forma predominante de energia e T2 ração com parte da energia complementada com óleo vegetal) e dez repetições. As dietas experimentais utilizadas se encontram descritas na tabela 1. A digestibilidade dos nutrientes foi avaliada dos 63 aos 71 dias de idade, onde foram quatro dias para adaptação a ração e a gaiola e quatro dias para a coleta das fezes. As rações e a água foram fornecidas à vontade durante as duas fases do experimento.

As fezes de cada animal foram coletadas em sua totalidade, uma vez ao dia, no período da manhã e acondicionadas em sacos plásticos e armazenadas em congelador a $-10^{\circ}$ C. Para realização das análises, as fezes de cada animal foram descongeladas a temperatura ambiente, pesadas, homogeneizadas e submetidas à présecagem a $55{ }^{\circ} \mathrm{C}$, durante 72 horas. As amostras foram levadas à estufa de ventilação forçada a $55^{\circ} \mathrm{C}$ durante 72 horas e posteriormente, retiradas e colocadas à temperatura e umidade ambiente durante 2 horas para pesagem e determinação da matéria pré-seca. Em seguida, as amostras foram processadas em moinho com peneira de $1 \mathrm{~mm}$. Foi avaliado o conteúdo \% da matéria seca (MS), da matéria orgânica $(\mathrm{MO})$, da proteína bruta $(\mathrm{PB})$, do Extrato Etéreo (EE) e da energia bruta (EB). Para as analises do conteúdo \% em PB e do EE, as amostras foram submetidas a uma prévia hidrólise ácida. Em seguida, foram calculados os coeficientes de digestibilidade aparente de (CDMS), (CDMO), (CDPB), (CDEE) e (CDEB) das dietas experimentais. As análises foram realizadas no Laboratório de Nutrição Animal do Departamento de Zootecnia da EV-UFMG, e seguiram a metodologia sugerida pelo Compêndio brasileiro de alimentação animal (1998), excetuando a energia bruta, para a qual foi utilizada a bomba adiabática de Parr. Para 
obtenção dos coeficientes de digestibilidade aparente (\%) da matéria seca (CDMS), matéria orgânica (CDMO), proteína bruta (CDPB), do extrato etéreo (CDEE) e da energia bruta (CDEB) das dietas. Utilizou-se a equação sugerida por Schneider and Flatt (1975):

CD do Nutriente $(\%)=$ Nutriente ingerido $(\mathrm{g})$ Nutriente das fezes (g) x100/Nutriente ingerido (g) .

O consumo médio diário (CMD) foi obtido pela pesagem da ração no inicio e ao final da fase de adaptação. E também das sobras ao final do teste.

CMD $(\mathrm{g})=$ Consumo $(\mathrm{g})-($ sobra + desperdício $)$ (g).

Foi avaliada a produção de cecotrófos durante 24 horas, no intuito de se conhecer a produção total de cecotrófos e sua contribuição nutricional para o animal alimentado com as diferentes dietas estudadas. Com o objetivo de se evitar a cecotrofia, cada animal recebeu um colar circular de madeira leve, medindo $25 \mathrm{~cm}$ de diâmetro, com um orifício central de $7 \mathrm{~cm}$ de diâmetro. Durante esse período, o consumo de ração foi controlado e os cecotrófos coletados de 2 em 2 horas para posterior plotagem dos dados em gráficos de dispersão com curva de tendência, registrando-se o coeficiente de determinação $\left(\mathrm{R}^{2}\right)$ de cada dieta.

Os cecotrófos de cada animal correspondentes ao período de coleta foram acondicionados em sacos plásticos hermeticamente fechados e congelados a $-18^{\circ} \mathrm{C}$ para posterior determinação das concentrações de MS, PB, EE e cinzas. As análises seguiram a metodologia sugerida pelo Compêndio Brasileiro de Alimentação Animal (1998). Para realização das análises, os cecotrófos foram descongelados à temperatura ambiente e homogeneizados. As amostras foram levadas à estufa de ventilação forçada a $55^{\circ} \mathrm{C}$ durante 72 horas e posteriormente, retiradas e colocadas à temperatura e umidade ambiente durante 2 horas para pesagem e determinação da matéria pré-seca. Sequencialmente, foi processada em moinho com peneira de $1,0 \mathrm{~mm}$. O material foi armazenado em frascos para posterior análise dos teores de MS, $\mathrm{PB}$ e EE.

Para as análises do conteúdo \% em PB e do EE, as amostras foram submetidas a uma prévia hidrólise ácida. A partir dos valores de composição química dos cecotrófos e da respectiva quantidade diária produzida, foi calculado o valor de contribuição de cada princípio nutritivo (MS, PB e EE) em relação ao consumo médio de ração desses mesmos princípios nutritivos em cada uma das dietas experimentais, utilizando-se as seguintes fórmulas (Carabaño et al., 1989).

$$
\mathrm{CN}(\mathrm{N}) \%=(\mathrm{A} \times 100) /(\mathrm{A}+\mathrm{B}) \text {. }
$$

Onde: $\mathrm{CN}(\mathrm{N})=$ Contribuição nutritiva dos cecotrófos do (nutriente) (\%);

$\mathrm{A}=$ Excreção do nutriente nos cecotrófos (g MS/dia);

$\mathrm{B}=$ Média de ingestão do (nutriente) durante o período experimental (g MS/dia).

Tabela 1. Dietas experimentais e sua composição química

\begin{tabular}{|c|c|c|}
\hline$\underline{\text { Ingredientes }}$ & Sem óleo & Com óleo \\
\hline Milho Farelo & 25,000 & 0,000 \\
\hline Soja Farelo 45\% & 23,008 & 16,792 \\
\hline Soja Casquinha & 20,521 & 6,072 \\
\hline Mdps & 15,789 & 12,558 \\
\hline Alfafa Feno & 10,000 & 30,878 \\
\hline Trigo Farelo & 0,000 & 23,359 \\
\hline Melaço Pó & 3,000 & 2,000 \\
\hline Fosfato Bicálcico & 0,704 & 0,443 \\
\hline Sal Comum & 0,500 & 0,500 \\
\hline Bentonita & 0,500 & 0,500 \\
\hline Vitaminas-Premix & 0,300 & 0,300 \\
\hline Calcário & 0,264 & 0,287 \\
\hline Minerais-Premix & 0,200 & 0,200 \\
\hline Dl-Metionina & 0,178 & 0,132 \\
\hline L-Lisina Hcl & 0,050 & 0,000 \\
\hline Oleo de Soja & 0,000 & 6,000 \\
\hline Total & 100,000 & 100,000 \\
\hline \multicolumn{3}{|c|}{ Composições Nutricionais calculadas (Matéria Natural) } \\
\hline Nutrientes & Qtde. & Qtde. \\
\hline Amido $(\%)$ & 24,681 & 14,000 \\
\hline Cálcio (\%) & 0,600 & 0,700 \\
\hline Energia (Mcal/Kg) & 2,705 & 2,700 \\
\hline Extrato Etéreo (\%) & 2,215 & 7,870 \\
\hline $\operatorname{FDA}(\%)$ & 17,500 & 19,189 \\
\hline FDN (\%) & 27,861 & 34,033 \\
\hline Fosforo Total (\%) & 0,400 & 0,500 \\
\hline Lisina Total (\%) & 0,800 & 0,800 \\
\hline Met.+Cist. Total (\%) & 0,600 & 0,600 \\
\hline Proteína Bruta $(\%)$ & 18,500 & 18,500 \\
\hline \multicolumn{3}{|c|}{ Composições nutricionais analisadas } \\
\hline Energia bruta $(\mathrm{Mcal} / \mathrm{Kg})$ & 3,940 & 4,458 \\
\hline Extrato Etéreo (\%) & 4,604 & 11,086 \\
\hline FDN (\%) & 24,726 & 31,090 \\
\hline $\mathrm{PB}(\%)$ & 18,774 & 19,154 \\
\hline
\end{tabular}

Os resultados foram submetidos à análise de variância mediante o programa estatístico $\underline{\mathrm{SAS}}$ (2004). 
FDN, FDA, fibra em detergente neutro, fibra em detergente acido, de acordo com o método de Van Soest et al. (1991).

\section{Resultados e Discussão}

Os resultados obtidos com os ensaios de digestibilidade e cecotrofia se encontram descritos nas tabelas 2,3 e 4 .

Tabela 2. Resultados obtidos com o ensaio de digestibilidade: Consumo médio diário (CMD em gramas), Excreção média diária (EXC em gramas), Coeficientes de Digestibilidade Aparente da matéria seca (CDMS), Matéria Orgânica (CDMO), Proteína Bruta (CDPB), do Extrato Etéreo (CDEE) e da Energia Bruta (CDEB) das dietas.

\begin{tabular}{lccc}
\hline & \multicolumn{2}{c}{ Tratamentos } & \\
\cline { 2 - 3 } Parâmetros & Sem óleo & Com óleo & CV (\%) \\
\hline PI, g & 2529 & 2510 & 2,07 \\
CMD, g & 147,95 & 147,57 & 8,15 \\
EXC, g & 56,77 & 58,88 & 12,14 \\
MS, \% & $60,95 \mathrm{~b}$ & $64,79 \mathrm{a}$ & 6,22 \\
MO, \% & 61,78 & 65,48 & 6,19 \\
PB, \% & 78,18 & 75,61 & 17,02 \\
EE, \% & 76,46 & 76,95 & 9,40 \\
EB, \% & $61,34 \mathrm{a}$ & $51,48 \mathrm{~b}$ & 8,29 \\
\hline
\end{tabular}

Médias seguidas de letras distintas na mesma linha diferem entre si pelo teste de Tukey a 5\%. CV: Coeficiente de variação

As dietas experimentais não ocasionaram diferenças significativas no CMD, EXC, CDMO, CDPB e CDEE. Com esses resultados pode-se dizer que a complementação lipídica não afetou a ingestão de alimento pelos animais, assim como não afetou a taxa de passagem dos nutrientes, que tende a diminuir com a inclusão de lipídios a dieta, ainda que tenha ocorrido um ligeiro aumento na CDMS.

De acordo com De Blas and Wiseman (2010), quando se adiciona lipídios à dieta, a tendência é a redução no consumo dos animais devido ao regulamento quimiostático do apetite. Falcão et al. (1996) também mencionam uma diminuição de ingestão da matéria seca em dietas com maiores percentuais de lipídios devido ao menor trânsito da digesta o que consequentemente, leva a aumento da eficiência da digestão. Entretanto nesse trabalho isso não foi observado, talvez como consequência da tentativa de manter os valores de energia das dietas próximas durante a formulação das mesmas.

Ferreira et al. (2006) e Pérez et al. (1996) não observaram diferenças na CDMO, CDPB e na ingestão de MS, quando forneceram dietas com alto percentual lipídico para coelhos em crescimento.

Não foi observado efeito dos tratamentos sobre a CDEE; resultados diferentes deste foram encontrados por Ferreira et al. (2006), Arruda et al. (2000), Cheeke (1995), Lopes et al. (1997), Xiccato (1996), Cesari et al. (2010) que observaram uma melhor CDEE em dietas com maior percentual de lipídios. Por outro lado, Maertens et al. (1985), Falcão et al. (1996) observaram que quando a inclusão de lipídio é alta pode ocorrer diminuição da CDEE, provavelmente porque a eficiência digestiva e atividade da microflora no ceco são negativamente afetados pelo excesso de lipídios.

Foi encontrado efeito significativo dos tratamentos sobre a CDE, sendo que a dieta sem adição de óleo vegetal apresentou melhor resultado $61,3 \%$ vs $51,5 \%$ da dieta complementada com lipídios, isso demonstra que pode ter havido um excesso de energia na forma de lipídios na dieta e essa foi perdida nas fezes ou ainda que esses animais utilizam melhor a energia dos carboidratos, pois partes dos mesmos podem ser fermentados no ceco e incorporado aos cecotrófos, que serão posteriormente reingeridos.

As curvas de cecotrofia objetivam avaliar o período de ocorrência dos cecotrófos e se houve alguma modificação causada pelas dietas no comportamento animal relativo à cecotrofia. Os gráficos correspondentes a cada tratamento estão demonstrados a seguir

O coeficiente de determinação $\left(R^{2}\right)$ visa avaliar a capacidade de um modelo matemático se adaptar em relação ao fenômeno observado. A partir disso pode-se constatar que o gráfico 2 no qual se tem um $\mathrm{R}^{2}$ de 0,687 vs 0,6011 o $\mathrm{R}^{2}$ encontrado no gráfico 1 explica melhor a possível interferência das dietas no fenômeno de cecotrofia realizado pelos coelhos.

Tabela 3. Composição química dos cecotrófos de acordo com as dietas experimentais $(\mathrm{g} / \mathrm{kg})$

\begin{tabular}{lccc}
\hline & \multicolumn{3}{c}{ Tratamentos } \\
\cline { 2 - 4 } Princípio nutritivo, \% & Sem óleo & Com óleo & CV, \% \\
\hline MS & 62,87 & 57,16 & 12,00 \\
PB & 30,70 & 29,20 & 7,36 \\
MO & $90,45 \mathrm{a}$ & $88,72 \mathrm{~b}$ & 0,36 \\
EE & $3,05 \mathrm{~b}$ & $4,54 \mathrm{a}$ & 13,88 \\
\hline
\end{tabular}

Médias seguidas de letras distintas na mesma linha diferem entre si pelo teste de Tukey a 5\%. CV: Coeficiente de variação. 
De acordo com Bellier and Gidenne (1996) a cecotrofia ocorre principalmente durante o início do período da luz e o pico de produção de cecotrófos ocorre das 9 às 12 horas. $\mathrm{Na}$ visualização dos resultados no gráfico 1 pode-se notar que a dieta sem complementação de óleo vegetal, seguiu esse comportamento padrão dos coelhos mencionados na literatura com a produção de cecotrófo concentrada no período de 9 as 11 da manhã.

No gráfico 2 percebe-se que a dieta complementada com óleo vegetal fugiu um pouco a essa regra geral, uma vez que o pico de produção foi antecipado para as 5 da manhã. De Blas and Wiseman (2010) relata que a produção e ingestão de cecotrófos no coelho doméstico ocorrem durante a noite, regularmente ocorrendo aproximadamente até quatro horas após a última refeição e em coelhos selvagem que são de hábitos noturnos, alimentando-se, portanto à noite a cecotrofia ocorre durante o dia. Os resultados do gráfico 2 corroboram para com essa teoria, pois a produção dos cecotrófos teve início à noite (23 horas) com o pico de produção as 5 horas da manhã.

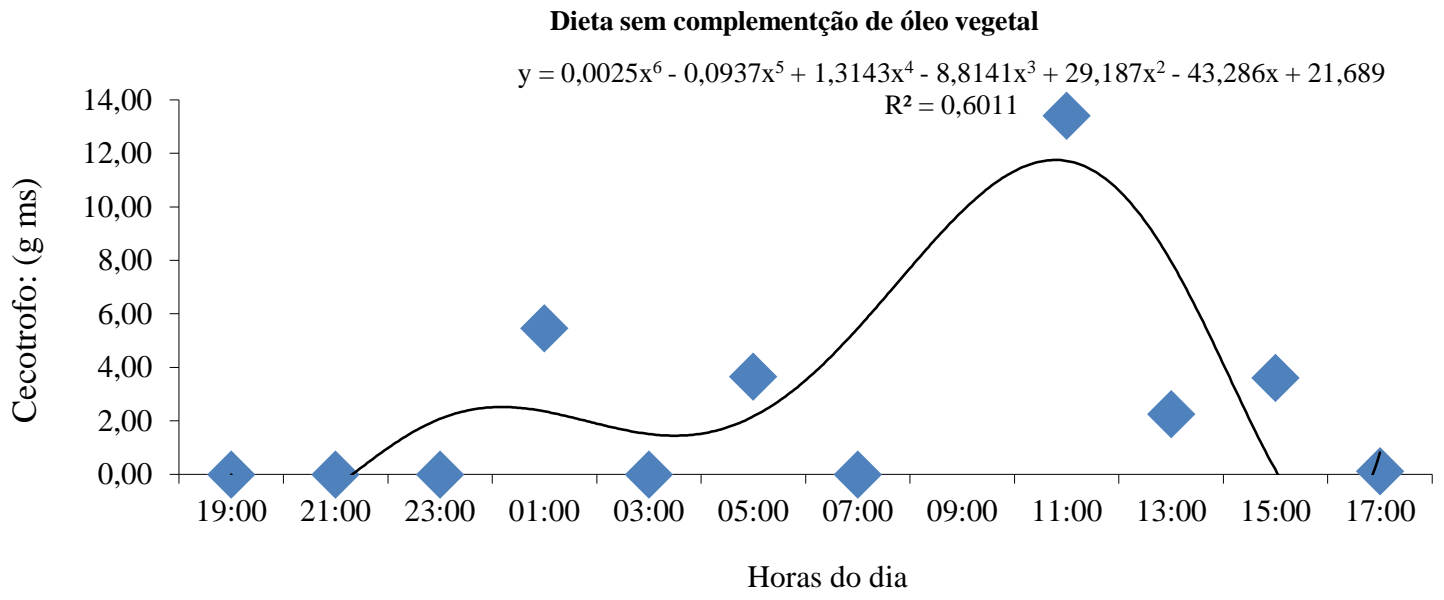

Gráfico 1. Produção de cecotrófos ao longo do dia, em coelhos com 70 dias de idade, alimentados com a dieta com carboidratos como principal fonte de energia.

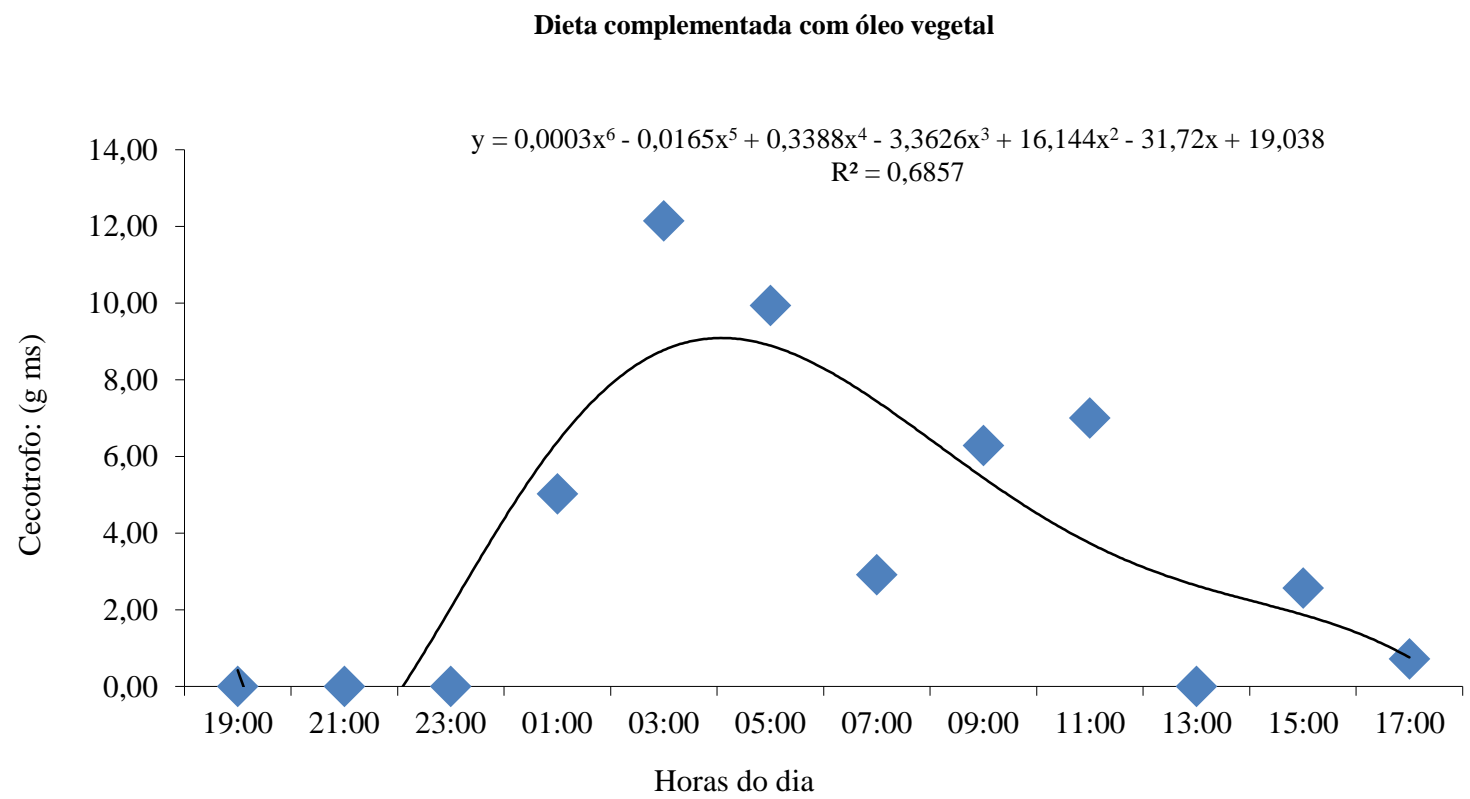

Gráfico 2. Produção de cecotrófos ao longo do dia, em coelhos com 70 dias de idade, alimentados com dieta complementada com lipídios. 
As dietas experimentais não influenciaram o conteúdo em MS e PB dos cecotrófos; entretanto influenciaram o conteúdo em MO e o EE, sendo que a dieta sem adição óleo vegetal proporcionou cecotrófos com maiores quantidades de $\mathrm{MO}$ $62,9 \%$ vs $57,2 \%$ da dieta complementada com óleo, assim é possível intuir que essa dieta ocasionou um maior processo fermentativo ocorrido no ceco, possibilitando uma proporção maior de compostos orgânicos em seus cecotrófos, concordando assim com as observações realizadas por Falcão et al. (1996) que os lipídios quando em alta concentrações na dieta, diminuem a atividade microbiana no ceco.

Tabela 4. Resultados obtidos com o ensaio de cecotrofia: Peso Médio Inicial, Produção de Cecotrófos (PC), Contribuição Nutritiva dos Cecotrófos em Matéria Seca (CNMS), Proteína Bruta Ingerida (PBI), Contribuição Nutritiva da Proteína Bruta (CNPB) e Contribuição Nutritiva do Extrato Etéreo (CNEE) de acordo com as dietas experimentais

\begin{tabular}{lccc}
\hline \multirow{2}{*}{ Parâmetros } & \multicolumn{2}{c}{ Tratamentos } & \\
\cline { 2 - 3 } PMI, kg & Sem óleo & Com óleo & CV $(\%)$ \\
PC g MS/dia & 2,510 & 2,475 & 6,25 \\
CNMS & 36,40 & 42,52 & 17,24 \\
PBI, g/dia & $17,83 \mathrm{~b}$ & $23,01 \mathrm{a}$ & 9,91 \\
CNPB & 27,94 & 25,12 & 12,70 \\
CNEE & 28,81 & 30,80 & 13,09 \\
\hline
\end{tabular}

Médias seguidas de letras distintas na mesma linha diferem entre si pelo teste de Tukey a 5\%. CV: Coeficiente de variação.

A proporção em EE nos cecotrófos foi mais elevada nos animais que receberam a dieta com complementação de óleo. É compreensível que os cecotrófos oriundos de dieta com um alto teor de lipídios, expressem o mesmo em sua constituição, uma vez que esses são o reflexo da dieta ingerida pelo animal.

Não houve efeitos dos tratamentos sobre PC, PBI, CNPB. Os dados de produção de cecotrófos encontrados nesse trabalho foram maiores que mencionados por Gidenne and Lebas (1984) e Herrera (2003), onde esses autores relatam que a produção media diária desse composto é de cerca de 20 a $25 \mathrm{~g} \mathrm{MS/dia.} \mathrm{A} \mathrm{dieta} \mathrm{com} \mathrm{maior} \mathrm{percentual}$ de lipídio apresentou um valor muito além desse, (42,5 g/MS/dia, não diferindo estatisticamente da dieta sem suplementação 36,4 g/MS/dia). A produção dos cecotrófos é condicionada a uma série de fatores e, sobretudo, pela composição da dieta, sendo esses produzidos em maior ou menor quantidade, o que se pode concluir que as dietas experimentais ofertadas estimularam os animais a produzirem uma maior quantidade desses que os avaliados pelos autores mencionados.

A dieta sem adição de óleo proporcionou uma maior CNEE de seus cecotrófos, isso vem a ser coerente uma vez que a dieta ofertada a esses animais possuíam um menor percentual de EE, quando comparados a dietas com complementação lipídica cujos cecotrófos também possuem um maior conteúdo em EE. Resultados semelhantes foram encontrados por Ferreira et al. (2006) onde as dietas com menor conteúdo lipídico também proporcionaram um CNEE maior $(13,6)$ o que é bem similar aos resultados obtidos nesse trabalho de 15,2 de CCEE da com carboidratos como principal fonte de energia.

\section{Conclusões}

Com os resultados obtidos através dos ensaios de digestibilidade e cecotrofia desenvolvidos, pode-se concluir que quando se adicionou óleo vegetal à dieta cunícola respeitando-se as recomendações de fibra e com valor de energia semelhante ao da dieta não complementada, não se observou diferenças no consumo e na digestibilidade dos nutrientes, excetuando-se os coeficientes de digestibilidade aparente da matéria seca e da energia bruta As mesmas também não causaram distúrbios na cecotrofia ou modificações na estrutura dos cecotrófos.

\section{Agradecimentos}

À Coordenação de Aperfeiçoamento Pessoal de Nível Superior - CAPES, pela concessão da bolsa de estudos.

\section{Referências Bibliográficas}

Arruda, A. M. V., Carregal, R. D. \& Ferreira, R. G. 2000. Desempenho produtivo e atividade microbiana cecal de coelhos alimentados com dietas contendo diferentes níveis de amido. Revista Brasileira de Zootecnia, 29, 762-768.

Bellier, R. \& Gidenne, T. 1996. Consequences of reduced fibre intake on digestion, rate of passage and caecal microbial activity in the young rabbit. British Journal of Nutrition, 75, 353-363.

Carabaño, R., Fraga, M. J. \& De Blas, J. C. 1989. Effect of protein source in fibrous diets on performance and digestive parameters of fattening rabbits. Journal of Applied Rabbit Research, 12, 201-204. 
Cesari, V., Grilli, G., Ferrazzi, V. \& Toschi, I. 2010. Influence of age at weaning and nutritive value of weaning diet on growth performance and caecal traits in rabbits. World Rabbit Science, 17, 195-205.

Cheeke, P. R. 1995. Alimentación y nutrición del conejo. Acribia, Zaragoza.

De Blas, C. \& Wiseman, J. 2010. Nutrition of the Rabbit. CABI, Cambridge.

Falcão, C. L., Bengala, F. J. P. \& Gonçalves, A. 1996. Effect of fat level and fiber nature on performances, digestibility, nitrogen balance and digestive organs in growing rabbits. 6th World Rabbit Congress. INRA, Toulouse.

Fernández-Carmona, J., Pascual, J. J. \& Cervera, C. 2000. The use of fat in rabbit diets. World Rabbit Science, 8, 29-56.

Ferreira, V. P. A., Ferreira, W. M., Saliba, E. O. S., Scapinello, C., Teixeira, A. O. B. F. \& Kamwa, E. B. 2006. Digestibilidade, cecotrofia, desempenho e rendimento de carcaça de coelhos em crescimento alimentados com rações contendo óleo vegetal ou gordura animal. Revista Brasileira de Zootecnia, 35, 1696-1704.

Fortun-Lamothe, L. 1997. Effects of dietary fat on reproductive performance of rabbit does: a review. World Rabbit Science, 5, 33-38.

Gidenne, T. \& Lebas, F. 1984. Evolution circadienne du contenu digestif chez le lapin en croissance: Relation avec la caecotrophie. World Rabbit Congress.

Herrera, A. 2003. Eficiência produtiva e avaliação nutricional de dietas simplificadas base de forragens para coelhos em crescimento. Escola de Veterinária. Universidade Federal de Minas Gerais, Belo Horizonte.

Jenkins, J. R. 1999. Feeding recommendations for the house rabbit. The Veterinary Clinics of North America. Exotic Animal Practice, 2, 143-151.

Lopes, D. C., Rostagno, H. S. \& Freitas, R. T. F. 1997. Valores de energia digestível de alimentos e digestibilidade dos nutrientes de rações com diferentes níveis de energia para coelhos em crescimento. Revista Brasileira de Zootecnia, 26, 1147-1152.
Maertens, L. 1998. Fats in rabbit nutrition: a review. World Rabbit Science, 6, 341-348.

Maertens, L., Huyghebaert, G. \& De Groote, G. 1985. Digestibility and digestible energy content of various fats for growing rabbits. Cuniculture Sciences, 3, 7-14.

Pascual, J. J., Tolosa, C., Cervera, C., Blas, E. \& Fernández-Carmona, J. 1999. Effect of diets with different digestible energy content on the performance of rabbit does. Animal Feed Science and Technology, 81, 105-117.

Pérez, J. M., Fortun-Lamothe, L. \& Lebas, F. 1996. Comparative digestibility of nutrients in growing rabbits and breeding does. Proceeding 6th World Rabbit Congress. INRA, Toulouse.

SAS. 2004. SAS/STAT User guide, Version 9.1.2. SAS Institute Inc, Cary, NC, USA.

Schneider, B. H. \& Flatt, W. P. 1975. The evaluation of feeds through digestibility experiments. University of Georgia Press, USA.

Van Soest, P. J., Robertson, J. B. \& Lewis, B. A. 1991. Methods for dietary fiber, neutral detergent fiber, and nonstarch polysaccharides in relation to animal nutrition. Journal of Dairy Science, 74, 3583-3597.

Xiccato, G. 1996. Nutrition of lactating does. Proceedings of the 6th World Rabbit Congress. INRA, Toulouse.

Xiccato, G. 1999. Feeding and meat quality in rabbits: a review. World Rabbit Science, 7, 7586.

\section{Article History:}

Received 9 December 2016

Accepted 2 January 2017

Available on line 4 February 2017

License information: This is an open-access article distributed under the terms of the Creative Commons Attribution License 4.0, which permits unrestricted use, distribution, and reproduction in any medium, provided the original work is properly cited 